\title{
Insulin resistance and adipokines serum levels in a caucasian cohort of hiv-positive patients undergoing antiretroviral therapy: a cross sectional study
}

Victoria Arama ${ }^{1,2}$, Catalin Tiliscan ${ }^{1,2^{*}}$, Adrian Streinu-Cercel ${ }^{1,2}$, Daniela lon ${ }^{1}$, Raluca Mihailescu ${ }^{2}$, Daniela Munteanu ${ }^{2}$, Adriana Hristea ${ }^{1,2}$, Stefan Sorin Arama' ${ }^{1}$ and the SLD-ART study group

\begin{abstract}
Background: Insulin resistance is frequent in human immunodeficiency virus (HIV) infection and may be related to antiretroviral therapy. Cytokines secreted by adipose tissue (adipokines) are linked to insulin sensitivity. The present study is aimed to assess the prevalence of insulin resistance (IR) and its association with several adipokines, in a non-diabetic Romanian cohort of men and women with HIV-1 infection, undergoing combination antiretroviral therapy (CART).

Methods: A cross-sectional study was conducted in an unselected sample of 89 HIV-1-positive, non-diabetic patients undergoing stable CART for at least 6 months. Metabolic parameters were measured, including fasting plasma insulin, and circulating adiponectin, leptin, resistin, tumor necrosis factor alpha (TNF-alpha) and interleukin-6 (IL-6) levels. Insulin resistance was estimated by measuring the Quantitative Insulin Sensitivity Check Index (QUICKI), using a cut-off value of 0.33. A linear regression model was fitted to QUICKI to test the association of IR and adipokines levels.

Results: A total of 89 patients (aged 18-65, median: 28 years) including 51 men (57.3\%) and 38 women (42.7\%) were included in the study. Fifty nine patients (66.3\%) were diagnosed with IR based on QUICKI values lower than the cut-off point. IR prevalence was $72.5 \%$ in men and $57.6 \%$ in women. The presence of the IR was not influenced by either the time of the HIV diagnosis or by the duration of CART. Decreased adiponectin and increased serum triglycerides were associated with increased $I R$ in men $(R=0.43, p=0.007)$. Hyperleptinemia in women was demonstrated to be associated with the presence of $\operatorname{IR}(R=0.33, p=0.03)$.

Conclusions: Given the significant prevalence of the IR in our young non-diabetic cohort with HIV infection undergoing antiretroviral therapy reported in our study and the consecutive risk of diabetes and cardiovascular events, we suggest that the IR management should be a central component of HIV-infection therapeutic strategy. As adipokines play major roles in regulating glucose homeostasis with levels varying according to the sex, we suggest that further studies investigating adipokines should base their analyses on gender differences.
\end{abstract}

Keywords: Adipokines, Adiponectin, Leptin, Antiretroviral therapy, Insulin resistance, HIV

\footnotetext{
* Correspondence: catalin_tiliscan@yahoo.com

${ }^{1}$ Carol Davila University of Medicine and Pharmacy, Bucharest, Romania

${ }^{2}$ Prof. Dr. Matei Bals National Institute of Infectious Diseases, Bucharest,

Romania
} 


\section{Background}

The introduction of combination antiretroviral therapy (cART) has significantly changed the course of human immunodeficiency virus (HIV) infection, increasing life expectancy but also leading to complex clinical and metabolic changes [1]. The term lipodystrophy was initially used to summarize these clinical and metabolic modifications [2]. The most important metabolic abnormalities observed in HIV-patients during cART include: insulin resistance (IR) with hyperinsulinemia and glucose intolerance [3], hypercholesterolemia with low levels of HDL-cholesterol, hypertriglyceridemia, lactic acidemia and hypercoagulopathy [4].

Lipodystrophy also associates fat redistribution: subcutaneous lipid stores wasting (Bichat's fat pad, buttocks and extremities), central adiposity and fat accumulation in the dorsocervical region (lipohypertrophy) [2]. As patients with these metabolic abnormalities have relatively high rates of experiencing a cardiovascular event, there is growing concern about the potential damaging impact of cART in HIV-positive patients. Metabolic abnormalities may result from various and combined effects of cART antiretroviral drugs. Several studies have reported that almost all protease inhibitors (PIs) can cause IR, effects likely caused by the inhibition of glucose transport mediated by glucose transporter type 4 (GLUT-4) $[5,6]$. Antiretroviral drugs may also impair endocrine functions of adipocytes [7]. Adipose tissue synthesizes various adipokines and hormones, such as adiponectin, leptin, resistin, tumour necrosis factor-alpha $(\mathrm{TNF}-\alpha)$, interleukins and release free-fatty acids [8]. Adiponectin and leptin may be involved in fat distribution and insulin sensitivity in HIV-infected patients [9]. Nucleoside reverse transcriptase inhibitors (NRTIs) may impair the secretion of adiponectin and thus may cause IR [10].

The aim of the study was to evaluate the prevalence of the IR and the association of the IR with clinical and biological variables, including serum adipokines, in a Caucasian HIV-infected non-diabetic population of men and women, undergoing cART.

\section{Methods}

A cross-sectional research was performed in a Caucasian cohort of HIV-1-positive patients attending Prof. Dr. Matei Bals National Institute of Infectious Diseases from Bucharest, between June 2009 and June 2010. The protocol was approved by the Prof. Dr. Matei Bals National Institute of Infectious Diseases ethics committee (protocol no. 190/15.01.2009). An informed consent was obtained from each of the research subjects. The study was part of a national research grant (SLD-ART no. 62077/ 2008) on HIV infected patients. All consecutive nondiabetic patients who signed a written informed consent to participate in the study were enrolled. We included HIV-infected men and women, aged 18 years and over, undergoing stable cART for at least 6 months. The patients completed during the medical visit a standard questionnaire for demographic parameters and for personal and family medical histories. Body mass index (BMI) was defined according to WHO criteria [11]. Overweight was defined as a BMI $\geq 25 \mathrm{~kg} / \mathrm{m}^{2}$ and obesity as BMI $\geq 30 \mathrm{~kg} / \mathrm{m}^{2}$.

Blood samples were tested for: Adiponectin (BioSource ELISA, KAPME09), Resistin (BioSource ELISA, KAPME 50), Leptin (BioSource EASIA KAP2281), Tumor necrosis factor alpha - TNF-alpha (BioSource EASIA KAP 1751), Interleukin-6 (IL-6) (BioSource EASIA KAP 1261), CD4 T cells count (BD FACSCanto II flow cytometer), HIV viral load (Roche LightCycler ${ }^{\circledR} 480$ Real-Time PCR System), fasting triglycerides, total cholesterol, low-density lipoprotein (LDL) cholesterol, high-density lipoprotein (HDL) cholesterol and fasting plasma glucose (dry slide technology, Vitros 950, Johnson \& Johnson), fasting insulin (ELx50 Bioelisa Washer BioTek).

IR was determined using the Quantitative Insulin Sensitivity Check Index (QUICKI), calculated as $1 /(\log$ (fasting insulin $\mu U / m L)+\log ($ fasting glucose $m g / d L$ ) [12].

In normal populations, mean QUICKI levels range from 0.37 to 0.39 [13]. Consistent with previous studies, QUICKI values were dichotomized at the cut-off of 0.33 , lower levels signifying IR [13-15].

We also assessed the homeostatic model assessment insulin resistance (HOMA-IR) index, first described by Matthews et al. in 1985 [16]. The formula used to calculate HOMA-IR was:

\section{HOMA-IR = fasting insulin $(\mu U / m L) \times$ fasting plasma} glucose $(\mathrm{mmol} / \mathrm{L}) / 22$.

We assumed a HOMA-IR value of $>3$ to define IR, consistent with previous studies investigating insulin resistance in Caucasian HIV populations [17].

\section{Statistical analyses}

In order to test whether the samples came from normally distributed populations we used the Shapiro-Wilk test. Variables with gaussian distribution were expressed as means (+/-standard deviation [SD]). For non-normally distributed continuous data results were presented as median (interquartile range). Differences in means between groups were tested using independent-sample t-tests. We calculated the $95 \%$ confidence interval for the difference between the population means. Mann-Whitney test for non parametric continuous variables, Chi-square or Fisher's exact test for categorical variables were used to test for statistical significance. Linear regression models were fitted to QUICKI score to test the association of IR with normally distributed continuous variables, included in the multivariate models either because of their known or suspected association with IR or based on an observed univariate association with QUICKI. Serum triglycerides, 
adiponectin, leptin, resistin, IL-6, TNF-alpha values were natural $\log$ transformed for the linear regression analysis. The correlation coefficient (R) was calculated to assess the linear correlation between the observed and modelpredicted values of the dependent variables. We computed two-tailed p-values. Statistical significance was set at 5\%, and all analyses were performed with SPSS $^{\circledR}$ Statistics v.17.0, IBM ${ }^{\circledR}$, New York, USA.

\section{Results and discussion}

A total of 89 patients (age 18-65, median: 28 years) including 51 men (57.3\%, age range: $18-63$ years, median: 32 years) and 38 women (42.7\% age range: $19-65$ years, median: 21 years) were included in the study. The mean BMI was $23.6( \pm 3.9) \mathrm{kg} / \mathrm{m}^{2}$. The median time from HIV diagnosis was 80.5 months and the median time on antiretroviral therapy was 72.5 months. Sixty five patients $(73 \%)$ had a current PI-based cART regimen. Median serum levels of adiponectin, leptin, resistin,
TNF alpha and IL-6 were $10.2 \mu \mathrm{g} / \mathrm{mL}, 1.4 \mathrm{ng} / \mathrm{mL}$, $5.5 \mathrm{ng} / \mathrm{mL}, 10.3 \mathrm{pg} / \mathrm{mL}$ and $26.5 \mathrm{pg} / \mathrm{mL}$, respectively.

Fifty nine patients (66.3\%) were diagnosed with IR based on QUICKI values lower than the cut-off point. The overall prevalence of IR was $62.9 \%$, based on HOMA-IR dichotomization. The median value of HOMA-IR was 3.77 (3.62). The patients were divided into Group 1 (patients without IR) and Group 2 (patients with IR). Group 1 patients had a mean QUICKI value of $0.35( \pm 0.02)$ and a median HOMA-IR of $1.84(0.67)$ vs. 0.29 $( \pm 0.02)$ and 4.62 (3.48) in Group 2, respectively. The subject characteristics stratified by IR status are shown in Table 1. Significant differences were noted in total cholesterol, triglycerides and adiponectin serum levels between the two groups. The prevalence of IR was not significantly different between men and women. No significant differences were found between Group 1 and Group 2 in age, BMI, total time on cART or current protease inhibitor treatment.

Table 1 Clinical and biological characteristics of study patients by IR

\begin{tabular}{|c|c|c|c|c|}
\hline Characteristics & Total patients & Patients without IR (Group 1) & Patients with IR (Group 2) & $P$ \\
\hline$N$ & 89 & $30(33.7)$ & $59(66.3)$ & \\
\hline Age (years) ${ }^{a}$ & $28(22)$ & $20.5(13)$ & $33(25)$ & 0.12 \\
\hline Males & $51(57.3)$ & $14(46.7)$ & $37(62.7)$ & 0.14 \\
\hline BMI $\left(\mathrm{kg} / \mathrm{m}^{2}\right)$ & $23.6 \pm 3.9$ & $22.6 \pm 3.8$ & $24.2 \pm 3.8$ & 0.3 \\
\hline Fasting glucose (mg/dl) & $85.7 \pm 13.7$ & $82.3 \pm 8.6$ & $87.2 \pm 15$ & 0.12 \\
\hline Haemoglobin A1c (\%) & $4.85 \pm 0.39$ & $4.82 \pm 0.25$ & $4.86 \pm 0.43$ & 0.66 \\
\hline Hypertension ${ }^{b}$ & $23(25.8)$ & $8(26.7)$ & $15(25.4)$ & 1.00 \\
\hline CD4 Tcell (cells $\left./ \mathrm{mm}^{3}\right)^{a}$ & $440(402)$ & $525(470)$ & $428(370)$ & 0.38 \\
\hline Patients with undetectable HIV viral load & $65(73)$ & $20(66.7)$ & $45(76.3)$ & 0.33 \\
\hline Time on antiretroviral therapy (months) ${ }^{a}$ & $72.5(95)$ & $80(120)$ & $70.5(95)$ & 0.21 \\
\hline Time from HIV diagnosis (months) ${ }^{a}$ & $80.5(83)$ & $92(96)$ & $76(64)$ & 0.36 \\
\hline Current Protease Inhibitor treatment & $65(73)$ & $23(76.7)$ & $42(71.2)$ & 0.58 \\
\hline Total cholesterol (mg/dl) & $192 \pm 38.4$ & $181.9 \pm 33$ & $199.6 \pm 39.9$ & $0.04^{*}$ \\
\hline HDL cholesterol (mg/dl) & $43.9 \pm 12.4$ & $42.9 \pm 10.5$ & $44.3 \pm 13.3$ & 0.53 \\
\hline LDL cholesterol (mg/dl) & $111.87 \pm 35.4$ & $107.3 \pm 32.7$ & $114.1 \pm 36.8$ & 0.39 \\
\hline Triglycerides $(\mathrm{mg} / \mathrm{dl})^{a}$ & $166(129)$ & $151.5(79)$ & $181(169)$ & $0.04^{*}$ \\
\hline Dyslipidemia $^{c}$ & $71(79.8)$ & $24(80)$ & $47(79.7)$ & 1.00 \\
\hline Hypertriglyceridemia $^{d}$ & $54(60.7)$ & $16(53.3)$ & $38(64.4)$ & 0.36 \\
\hline Adiponectin $(\mu \mathrm{g} / \mathrm{mL})^{a}$ & $10.2(12.5)$ & $16(28.8)$ & $9.4(10.7)$ & $0.00^{*}$ \\
\hline Leptin $(\mathrm{ng} / \mathrm{mL})^{\text {a }}$ & $1.4(3.6)$ & $1.8(2.9)$ & $1.3(4.1)$ & 0.53 \\
\hline Resistin $(\mathrm{ng} / \mathrm{mL})^{a}$ & $5.5(3.6)$ & $5.2(5.1)$ & $5.7(3.4)$ & 0.63 \\
\hline TNF alfa $(\mathrm{pg} / \mathrm{mL})^{\mathrm{a}}$ & $10.3(15.1)$ & $11.9(16.2)$ & $9.7(15)$ & 0.62 \\
\hline $\mathrm{IL}-6(p g / m L)^{a}$ & $26.5(10.3)$ & $26.6(23.1)$ & $26.5(8.4)$ & 0.52 \\
\hline
\end{tabular}

Data are shown as mean \pm SD or number (percent).

${ }^{a}$ Results presented as median (interquartile range).

b Hypertension defined as blood pressure $\geq 130 / \geq 85 \mathrm{~mm} \mathrm{Hg}$ or antihypertensive medication.

c Dyslipidemia defined as elevation of one of the following: total cholesterol, LDL cholesterol, triglyceride serum concentrations or a decrease of HDL cholesterol.

d Hypertriglyceridemia defined as serum triglycerides $\geq 150 \mathrm{mg} / \mathrm{dl}$.

* p $<0.05$ Group 1 vs. Group 2.

$\mathrm{p}$ values based on $t$-test for equality of means or Mann-Whitney test for continuous variables, Chi-square or Fisher's exact test for categorical variables. 
In order to determine the relationship between laboratory parameters and IR, first we assessed the relationship of all individual variables with QUICKI score by fitting a linear regression model. The variables with univariate association to QUICKI were further tested to improve the model predictivity. After all variables were tested in the univariate analysis for the entire population, including BMI and age, only log transformed triglycerides serum levels (LOGTriglycerides) were significantly associated with QUICKI ( $\mathrm{R}=0.24$, $\mathrm{p}=0.01)$.

Fifity one men were enrolled in the study and 37 (72.5\%) were diagnosed with IR. Male subject characteristics stratified by IR status are shown in Table 2. Significant betweengroup differences persisted in triglycerides and adiponectin serum levels. The significant variables selected to build the additive linear regression model in men, the estimate terms for the model and corresponding test statistics are provided in Table 3. Of the variables tested only log transformed adiponectin (LOGAdiponectin) and LOGTriglycerides had a significant effect on QUICKI values in our cohort of male subjects. Taken together, the analysis indicates that LOGAdiponectin with LOGTriglycerides were associated with QUICKI index $(\mathrm{R}=0.43, \mathrm{p}=0.007)$ in males. The additive models that included age and BMI together with LOGAdiponectin and LOGTriglycerides lacked statistical significance.

Of 38 women enrolled 22 (57.6\%) were diagnosed with IR. Female laboratory characteristics stratified by IR status are shown in Table 2. Significant between-group differences were observed in HDL cholesterol and leptin serum levels. In women, of all variables tested in the univariate analysis, only log transformed leptin serum levels (LOGLeptin) were significantly associated with QUICKI $(\mathrm{R}=0.33, \mathrm{p}=0.03) \quad$ (Table 4). Other selected variables, including age and BMI, did not show any improvement of our additive model.

IR appears when maintaining normal physiological values of glycaemia require insulin levels that exceed normal concentrations [18]. IR is associated with the development of diabetes and obesity [19]. Up to $20 \%$ of non-HIV western population may have IR [20,21]. Several studies reported a two-fold increase of cardiovascular risk compared to non-insulin resistant controls $[22,23]$. In the pre-cART era several cross-sectional studies reported slightly increased [24] or normal [25] insulin sensitivity when compared to uninfected controls. cART introduction led to increases in fasting insulin and decreases in insulin sensitivity, effects dependent both on the class of the antiretroviral used and the different antiretrovirals within each class [26,27]. Different studies have evaluated the prevalence of IR at 20 to $50 \%$, in HIV-positive patients [28]. We noted higher levels of IR than reported by previous studies, independent of current treatment with PIs and of BMI, which may be attributed in part to our method of assessment. QUICKI has a very good correlation with the glucose clamp index of insulin sensitivity, which is considered the gold standard method for IR assessment [15]. IR presence in our population of HIV-positive young adults was not influenced either by the time from HIV diagnosis or to the duration of cART. This finding may suggest that the effect of cART on IR may not have a cumulative or long-term response, a similar result being reported also by other studies [29]. As IR presence is associated with an increased risk of diabetes, cardiovascular events, stroke and death, the reduction of its prevalence must be considered an essential therapeutic target in HIV-patients undergoing retroviral therapy.

Table 2 Biological characteristics of male and female study patients by IR

\begin{tabular}{|c|c|c|c|c|c|c|}
\hline \multirow[t]{2}{*}{ Characteristics } & \multicolumn{3}{|c|}{ Males } & \multicolumn{3}{|c|}{ Females } \\
\hline & $\begin{array}{c}\text { Patients without IR } \\
\text { (Group 1) }\end{array}$ & $\begin{array}{l}\text { Patients with IR } \\
\text { (Group 2) }\end{array}$ & $P$ & $\begin{array}{c}\text { Patients without IR } \\
\text { (Group 1) }\end{array}$ & $\begin{array}{l}\text { Patients with IR } \\
\text { (Group 2) }\end{array}$ & $P$ \\
\hline$N$ & $14(27.5)$ & $37(72.5)$ & & $16(42.1)$ & $22(57.6)$ & \\
\hline Adiponectin $(\mu \mathrm{g} / \mathrm{mL})^{a}$ & $14.1(31.8)$ & $8.3(10.5)$ & $0.03^{*}$ & $18.1(28.1)$ & $14.4(12.7)$ & 0.18 \\
\hline Leptin (ng/mL) ${ }^{a}$ & $0.6(1.2)$ & $0.9(1.2)$ & 0.25 & $2.8(2.3)$ & $5.3(8.8)$ & $0.04^{*}$ \\
\hline Resistin (ng/mL) ${ }^{a}$ & $4.9(3.3)$ & $5.5(3)$ & 0.8 & $5.3(6)$ & $6.2(4.6)$ & 0.5 \\
\hline TNF alpha $(\mathrm{pg} / \mathrm{mL})^{\mathrm{a}}$ & $13.8(17.6)$ & $10.4(14.6)$ & 0.65 & $10.4(14.3)$ & $8.9(14.8)$ & 0.62 \\
\hline IL-6 (pg/mL) ${ }^{a}$ & $35.8(22.6)$ & $27.7(18.5)$ & 0.46 & $23.9(14.4)$ & $25.2(4.9)$ & 0.22 \\
\hline Total cholesterol (mg/dl) & $179.5 \pm 33$ & $197.6 \pm 35.8$ & 0.1 & $184 \pm 34$ & $202.9 \pm 46.6$ & 0.17 \\
\hline LDL cholesterol (mg/dl) & $107.7 \pm 34$ & $115 \pm 37.2$ & 0.52 & $107 \pm 32.6$ & $112 \pm 36.9$ & 0.62 \\
\hline HDL cholesterol (mg/dl) & $42.2 \pm 12.5$ & $40 \pm 9.1$ & 0.19 & $41.7 \pm 8.5$ & $48 \pm 16$ & $0.02^{*}$ \\
\hline Triglycerides (mg/dl) ${ }^{a}$ & $117.5(84)$ & $217(157)$ & $0.01^{*}$ & $167.5(41)$ & $165.5(140)$ & 0.96 \\
\hline
\end{tabular}

Data are shown as mean \pm SD or number (percent).

${ }^{a}$ Results presented as median (interquartile range).

* $\mathrm{p}<0.05$ Group 1 vs. Group 2.

$\mathrm{p}$ values based on $t$-test for equality of means or Mann-Whitney test for continuous variables, Chi-square or Fisher's exact test for categorical variables. 
Table 3 Multivariate analysis: effect of selected variables (LOGAdiponectin, LOGTriglycerides) on QUICKI (dependent variable) in male subjects

\begin{tabular}{lcccc}
\hline Independent Variables & Unstandardized Coefficients (B) & Standardized Coefficients (beta) & $95 \%$ Confidence interval for B & P \\
\hline LOGAdiponectin $^{\text {b }}$ & 0.009 & 0.278 & $(0.000 ; 0.001)$ & 0.04 \\
LOGTriglycerides $^{b}$ & -0.013 & -0.261 & $(-0.026 ; 0.01)$ & 0.03 \\
\hline
\end{tabular}

${ }^{\mathrm{b}}$ LOGAdiponectin - log transformed adiponectin serum levels computed variable for linear regression.

${ }^{b}$ LOGTriglycerides - log transformed triglycerides serum levels computed variable for linear regression.

Adiponectin is exclusively secreted from adipose tissue and has an important role as a mediator of peroxisome proliferator-activated receptor gamma action [30]. Adiponectin may increase insulin responsiveness of the glucose transport system in adipocytes through increased GLUT4 gene expression and increased GLUT4 recruitment to the plasma membrane [31]. Several studies have reported that adipocyte differentiation was perturbed both in vitro and in vivo as a direct effect of cART and the consecutive decrease in serum adiponectin levels was associated with insulin resistance [32,33]. We found an inverse correlation between adiponectin and IR in men, but not in females which partially concurs with previous studies. Vigouroux et al. (2003) have also reported that adiponectin was positively correlated with insulin sensitivity in a cohort of 131 HIV-infected men with PI-based cART regimens [9]. In a study on 237 Afro-Caribbean HIV-1-infected patients, published in 2011, Deloumeaux et al. found also an inverse correlation between adiponectin and IR in both men and women. After adjusting for clinical and metabolic parameters, the correlation between adiponectin and insulin resistance was observed only in women [34], a conclusion that contradicts our results and the previously mentioned study. Alterations of serum adiponectin concentration in relation to insulin resistance was also observed in a cohort of HIV-infected youths (aged 5.0 - 19.4 years), in a study published by Viganò et al. in 2011, which did not take into account sex differences but evaluated lipodystrophy. Low adiponectin concentration was associated to central fat and mixed lipohypertrophy and to signs of IR [35]. Other studies have also shown that adiponectin was inversely associated with IR, type 2 diabetes and obesity in HIV and non HIV-patients in without adjusting for gender differences [36,37].

Leptin is a 167-amino-acid adipokine secreted mainly by white adipose tissue. The levels of leptin are positively correlated with the amount of body fat [37]. Leptin regulates energy homeostasis and various neuroendocrine functions [38]. Our data showed higher levels of leptin in women with IR vs women without IR and a weak correlation between IR presence and leptin, observed in the linear regression model, with QUICKI as dependent and LOGLeptin as independent variable. Leptin did not contribute to an improvement of our linear model of IR in men. Other studies investigating this association have reported inconclusive results but very few have based their analyses on gender differences. Azzoni et al. in 2010 observed that serum leptin levels are inversely associated with HIV replication, independent of disease progression. In the same study, the authors reported a weak association between leptin levels and the Homeostatic Model Assessment for Insulin Resistance (HOMA-IR) [39]. Mynarcik et al. (2002) found that levels of leptin did not correlate with insulin sensitivity, in HIV-infected subjects with body fat redistribution [37]. In other study investigating adipokines and antiretroviral therapy related lipodystrophy, Tao et al. (2009) observed that leptin was positively correlated with the levels of total body fat, limb fat, and trunk fat, both in lipodystrophic and patients without body fat redistribution, but did not correlate with IR, and might serve only as a biomarker for total body fat mass [40]. Nagy et al. (2003) found a significant association between leptin concentrations and IR in patients with different morphological phenotypes. The lowest mean leptin levels were observed in patients with lipoatrophic changes. Hypoleptinemia was independently associated with IR in patients with lipoatrophy. Highest leptin concentrations were observed in patients with lipohypertrophy. Leptin levels in between the two extremes were observed in subjects with no body shape changes [41]. The role of leptin alterations in IR pathogenesis remains to be clarified by future long-term and prospective studies.

Despite the relative low number of patients, our study has several strengths. This is the first study in Romania to investigate adipokine dysregulation in association with HIV metabolic abnormalities during cART. The young age of our cohort is a distinct feature for the HIV epidemiology in Romania, compared with western European countries.

Table 4 Univariate analysis: effect of selected variables (LOGLeptin) on QUICKI (dependent variable) in female subjects

\begin{tabular}{lcccc}
\hline Independent Variables & Unstandardized Coefficients (B) & Standardized Coefficients (beta) & $95 \%$ Confidence interval for B & P \\
\hline LOGLeptin $^{c}$ & -0.012 & -0.339 & $(-0.023 ; 0.000)$ & 0.03
\end{tabular}

cLOGLeptin - log transformed leptin serum levels computed variable for linear regression. 
The patients were probably infected parenterally in their childhood and adolescence between 1980-1990. QUICKI was used in order to diagnose IR, which is one of the most accurate surrogate indexes for determining insulin sensitivity in humans. Very few studies have evaluated untill now IR risk factors based on sex differences. Factors associated with IR, which have been lacking in previous studies, including the time from HIV diagnosis and the time on cART were measured and evaluated in our analyses.

Our study has some limitations. Correlations were small to moderate in magnitude. The association between IR and adiponectin/triglycerides in men and IR and leptin in women disappeared after including age and BMI in the regression models. This may be caused by BMI variations and changes in adiponectin with age that may contribute to IR pathogenesis and also by the low number of enrolled patients, with loss of statistical power as more variables were added. QUICKI was used to diagnose IR, a surrogate method, which has some limitations, such as the absence of a definite threshold for IR in Caucasian populations. QUICKI requires the quantification of fasting plasma insulin. Similar observations in clinical practice may be predicted with the use of International Diabetes Federation (IDF) or Adult Treatment Panel III (ATPIII) definitions of metabolic syndrome, which may provide cheaper ways for detecting patients at risk of diabetes and cardiovascular events. As leptin may be positively correlated with the levels of total body fat, limb fat, and trunk fat [41], the predictive value of our model might have been improved if we had included body fat redistribution in our analysis.

\section{Conclusions}

We report a significant prevalence of IR in a young nondiabetic Caucasian population with HIV infection undergoing antiretroviral therapy that was independent of the time of the HIV diagnosis. Hypoadiponectinemia in men and hyperleptinemia in women was demonstrated to be associated with IR presence which brings new data to support the role of these cytokines in IR pathogenesis. As adipokines play major roles in regulating glucose homeostasis with levels varying according to sex, we suggest that further studies investigating adipokines should base their analyses on gender differences. In addition, new studies are needed to investigate the mechanisms contributing to the significant differences observed between men and women.

SLD-ART Study Group: Sorin Petrea, Otilia Benea, Mariana Mărdărescu, Monica Luminos, Dan Oțelea, Ana Maria Tudor, Simona Paraschiv, Viorica Leoveanu (deceased), Mihaela Rădulescu, Mihai Lazăr, Sergiu Costoiu, loana Olaru, Cristina Popescu, Cristina Loredana Benea, Ruxandra Moroti, Violeta Molagic, Viorica Poghirc.

\section{Abbreviations}

CART: combined antiretroviral therapy; BMI: Body mass index; GLUT4: glucose transporter type 4; HDL: high-density lipoprotein; HIV: human immunodeficiency virus; IL-6: Interleukin-6; IR: insulin resistance; LDL: Iow- density lipoprotein; NRTIs: Nucleoside reverse transcriptase inhibitors; PIs: protease inhibitors; QUICKI: Quantitative Insulin Sensitivity Check Index; TNF-a: tumour necrosis factor-alpha.

\section{Competing interests}

The authors declare that they have no competing interests.

\section{Authors' contributions}

VA and ASC concieved the design of the study, coordinated the research study group and drafted the manuscript. CT and SSA performed statystical analyses and drafted the manuscript. RM and DM participated in patient enrollment and follow-up and reviewed the literature. AH and DI reviewed the paper before submission. VA, CT, ASC, DI, RM and SSA contributed equally to this work. All authors read and approved the final manuscript.

\section{Acknowledgements}

The authors thank all patients for their contribution to the research, as well as all the medical staff involved in this study, especially loana Olaru, Cristina Popescu, Cristina Loredana Benea, Anca Streinu Cercel and Ana Maria Tudor for their involvement in recruiting and in the follow-up of the patients and to Viorica Leoveanu (deceased) for performing the measurements for the adipocytokines. The study was funded by the National Authority for Scientific Research (Grant No. 62077/2008, 2008-2011). Coordinating center: Prof. Dr. Matei Bals National Institute of Infectious Diseases, Bucharest, Romania. Project Manager: Prof. Adrian Streinu Cercel; Scientific coordinator: Assoc. Prof. Victoria Arama.

Received: 27 June 2012 Accepted: 23 January 2013

Published: 26 January 2013

\section{References}

1. Falutz J: Management of Fat Accumulation in Patients with HIV Infection. Curr HIV/AIDS Rep 2011 Sep, 8(3):200-208.

2. Giralt $M$, Domingo P, Villarroya F: Adipose tissue biology and HIVinfection. Best Pract Res Clin Endocrinol Metab 2011 Jun, 25(3):487-499.

3. Feeney ER, Mallon PW: Insulin resistance in treated HIV infection. Best Pract Res Clin Endocrinol Metab 2011 Jun, 25(3):443-458.

4. Giannarelli C, Klein RS, Badimon JJ: Cardiovascular implications of HIV-induced dyslipidemia. Atherosclerosis 2011 Dec, 219(2):384-389.

5. Koster JC, Remedi MS, Qiu H, Nichols CG, Hruz PW: HIV protease inhibitors acutely impair glucose-stimulated insulin release. Diabetes $2003 \mathrm{Jul}$, 52(7):1695-1700.

6. Hui DY: Effects of HIV protease inhibitor therapy on lipid metabolism. Prog Lipid Res 2003 Mar, 42(2):81-92.

7. Chaparro J, Reeds DN, Wen W, Xueping E, Klein S, Semenkovich CF, Bae KT, Quirk EK, Powderly WG, Yarasheski KE, Li E: Alterations in thigh subcutaneous adipose tissue gene expression in protease inhibitorbased highly active antiretroviral therapy. Metabolism 2005 May, 54(5):561-567.

8. Rabe K, Lehrke M, Parhofer KG, Broedl UC: Adipokines and insulin resistance. Mol Med 2008, 14(11-12):51-741.

9. Vigouroux C, Maachi M, Nguyên TH, Coussieu C, Gharakhanian S, Funahashi T, Matsuzawa Y, Shimomura I, Rozenbaum W, Capeau J, Bastard JP: Serum adipocytokines are related to lipodystrophy and metabolic disorders in HIV-infected men under antiretroviral therapy. AIDS 2003, 17:1503-1511.

10. Jones SP, Qazi N, Morelese J, Lebrecht D, Sutinen J, Yki-Jărvinen H, Back DJ, Pirmohamed M, Gazzard BG, Walker UA, Moyle GJ: Assessment of adipokine expression and mitochondrial toxicity in HIV patients with lipoatrophy on stavudine- and zidovudine-containing regimens. J Acquir Immune Defic Syndr 2005 Dec 15, 40(5):565-572.

11. World Health Organization: Obesity: Preventing and Managing the Global Epidemic. Geneva: Technical Report Series No. 894. WHO; 2000.

12. Katz A, Nambi SS, Mather K, Baron AD, Follmann DA, Sullivan G, Quon MJ: Quantitative insulin sensitivity check index: a simple, accurate method for assessing insulin sensitivity in humans. J Clin Endocrinol Metab 2000 Jul, 85(7):2402-2410.

13. Castaneda-Sceppa C, Bermudez OI, Wanke C, Forrester JE: Predictors of insulin resistance among Hispanic adults infected with or at risk of infection with the human immunodeficiency virus and hepatitis $C$ virus. J Viral Hepat 2008 Dec, 15(12):878-887. 
14. Jones CY, Wilson IB, Greenberg AS, Shevitz A, Knox TA, Gorbach SL, Spiegelman D, Jacobson DL, Wanke C: Insulin resistance in HIV-infected men and women in the nutrition for healthy living cohort. $J$ Acquir Immune Defic Syndr 2005 Oct 1, 40(2):202-211.

15. Ascaso JF, Pardo S, Real JT, Lorente RI, Priego A, Carmena R: Diagnosing insulin resistance by simple quantitative methods in subjects with normal glucose metabolism. Diabetes Care 2003 Dec, 26(12):3320-3325.

16. Matthews DR, Hosker JP, Rudenski AS, Naylor BA, Treacher DF, Turner RC: Homeostasis model assessment: insulin resistance and beta-cell function from fasting plasma glucose and insulin concentrations in man. Diabetologia 1985 Jul, 28(7):412-419.

17. Matti A, Nasta P, Borghi F, Cologni G, Ricci A, Gatti F, Puoti M, Carosi G: Predictors of insulin resistance (IR) in HIV/HCV co-infected patients treated with pegylated interferon alpha 2-a (Peg/IFN) and ribavirin (RBV). Treatment and Prevention: 4th IAS Conference on HIV Pathogenesis; 2007:22-25.

18. Lebovitz HE: Insulin resistance: definition and consequences. Exp Clin Endocrinol Diabetes 2001, 109(Suppl. 2):S135-S148.

19. DeFronzo RA, Ferrannini E: Insulin resistance. A multifaceted syndrome responsible for NIDDM, obesity, hypertension, dyslipidemia, and atherosclerotic cardiovascular disease. Diabetes Care 1991, 14:173-194.

20. Ford ES, Giles WH, Dietz WH: Prevalence of the metabolic syndrome among US adults: findings from the third national health and nutrition examination survey. JAMA 2002, 287:356-359.

21. Marques-Vidal P, Mazoyer E, Bongard V, Gourdy P, Ruidavets JB, Drouet L, Ferrières J: Prevalence of insulin resistance syndrome in southwestern France and its relationship with inflammatory and hemostatic markers. Diabetes Care 2002, 25:1371-1377.

22. Pyörälä M, Miettinen $H$, Halonen $P$, Laakso M, Pyörälä K: Insulin resistance syndrome predicts the risk of coronary heart disease and stroke in healthy middle-aged men: the 22-year follow-up results of the Helsinki policemen study. Arterioscler Thromb Vasc Biol 2000, 20:538-544.

23. Hedblad B, Nilsson P, Engström G, Berglund G, Janzon L: Insulin resistance in non-diabetic subjects is associated with increased incidence of myocardial infarction and death. Diabet Med 2002, 19:470-475.

24. Hommes MJ, Romijn JA, Endert E, Eeftinck Schattenkerk JK, Sauerwein HP: Insulin sensitivity and insulin clearance in human immunodeficiency virus-infected men. Metabolism 1991 Jun, 40(6):651-656.

25. Walli R, Herfort O, Michl GM, Demant T, Jäger H, Dieterle C, Bogner JR, Landgraf R, Goebel FD: Treatment with protease inhibitors associated with peripheral insulin resistance and impaired oral glucose tolerance in HIV-1-infected patients. AIDS 1998, 12:F167-F173.

26. Dubé MP, Edmondson-Melançon H, Qian D, Aqeel R, Johnson D, Buchanan TA: Prospective evaluation of the effect of initiating indinavir-based therapy on insulin sensitivity and B-cell function in HIV-infected patients. J Acquir Immune Defic Syndr 2001, 27:130-134.

27. Yan Q, Hruz PW: Direct comparison of the acute in vivo effects of HIV protease inhibitors on peripheral glucose disposal. J Acquir Immune Defic Syndr 2005, 40:398-403.

28. Florescu D, Kotler DP: Insulin resistance, glucose intolerance and diabetes mellitus in HIV-infected patients. Antivir Ther 2007, 12(2):149-162.

29. De Wit S, Sabin CA, Weber R, Worm SW, Reiss P, Cazanave C, El-Sadr W, Monforte A, Fontas E, Law MG, Friis-Møller N, Phillips A: Incidence and risk factors for new-onset diabetes in HIV-infected patients: the Data Collection on Adverse Events of Anti-HIV Drugs (D:A:D) study. Diabetes Care 2008, 31(6):9-1224. Epub 2008 Feb 11.

30. Ziemke F, Mantzoros CS: Adiponectin in insulin resistance: lessons from translational research. Am J Clin Nutr 2010, 91(1):258S-261S.

31. Fu Y, Luo N, Klein RL, Garvey WT: Adiponectin promotes adipocyte differentiation, insulin sensitivity, and lipid accumulation. J Lipid Res 2005, 46(7):79-1369. Epub 2005 Apr 16.

32. Kubota N, Terauchi Y, Yamauchi T, Kubota T, Moroi M, Matsui J, Eto K, Yamashita T, Kamon J, Satoh H, Yano W, Froguel P, Nagai R, Kimura S, Kadowaki T, Noda T: Disruption of adiponectin causes insulin resistance and neointimal formation. J Biol Chem 2002, 277:25863-25866.

33. Weyer C, Funahashi T, Tanaka S, Hotta K, Matsuzawa Y, Pratley RE, Tataranni PA: Hypoadiponectinemia in obesity and type 2 diabetes: close association with insulin resistance and hyperinsulinemia. J Clin Endocrinol Metab 2001, 86:1930-1935.

34. Deloumeaux J, Maachi M, Sow-Goerger MT, Lamaury I, Velayoudom FL, Cheret A, Batard ML, Muller P, Bastard JP, Chene G, Capeau J, Foucan L: Adiponectin and leptin in Afro-Caribbean men and women with HIV infection: association with insulin resistance and type 2 diabetes. Diabetes Metab 2011 Apr, 37(2):98-104.

35. Viganò A, Zuccotti GV, Cerini C, Stucchi S, Puzzovio M, Giacomet V, Mora S: Lipodystrophy, Insulin Resistance, and Adiponectin Concentration in HIVInfected Children and Adolescents. Curr HIV Res 2011 Jul, 9(5):321-326.

36. Yamamoto $Y$, Hirose H, Saito I, Tomita M, Taniyama M, Matsubara K, Okazaki Y, Ishii T, Nishikai K, Saruta T: Correlation of the adipocyte-derived protein adiponectin with insulin resistance index and serum high-density lipoprotein-cholesterol, independent of body mass index, in the Japanese population. Clin Sci (Lond) 2002, 103:137-142.

37. Mynarcik DC, Combs T, McNurlan MA, Scherer PE, Komaroff E, Gelato MC: Adiponectin and leptin levels in HIV-Infected subjects withinsulin resistance and body fat redistribution. JAIDS 2002, 31:514-520.

38. Kelesidis T, Kelesidis I, Chou S, Mantzoros CS: Narrative review: the role of leptin in human physiology: emerging clinical applications. Ann Intern Med 2010 Jan 19, 152(2):93-100.

39. Azzoni L, Crowther NJ, Firnhaber C, et al: Association between HIV replication and serum leptin levels: an observational study of a cohort of HIV-1-infected South African women. J Int AIDS Soc 2010 Sep 7, 13:33.

40. Tao MM, Zhang L, Qiu ZF, Xie J, Han Y, Yu W, Li M, Li TS: Adipokines and highly active antiretroviral therapy related lipodystrophy: clinical study of 52 cases. Zhonghua Yi Xue Za Zhi 2009 Apr 7, 89(13):867-871.

41. Nagy GS, Tsiodras S, Martin LD, Avihingsanon A, Gavrila A, Hsu WC, Karchmer AW, Mantzoros CS: Human immunodeficiency virus type 1-related lipoatrophy and lipohypertrophy are associated with serum concentrations of leptin. Clin Infect Dis 2003, 36:795-802

doi:10.1186/1472-6823-13-4

Cite this article as: Arama et al:: Insulin resistance and adipokines serum levels in a caucasian cohort of hiv-positive patients undergoing antiretroviral therapy: a cross sectional study. BMC Endocrine Disorders 2013 13:4.

\section{Submit your next manuscript to BioMed Central and take full advantage of:}

- Convenient online submission

- Thorough peer review

- No space constraints or color figure charges

- Immediate publication on acceptance

- Inclusion in PubMed, CAS, Scopus and Google Scholar

- Research which is freely available for redistribution 